\title{
A New Approach to Computing
}

\author{
by \\ Nirmalya Basu \\ Date of Birth: May 3, 1984 \\ Qualification: Master of Engineering in Electronics and Telecommunication Engineering \\ Email: nirmalya1basu@gmail.com
}

\begin{abstract}
I have proposed here a new scheme of representing information which is that any physical quantity with a specific identity can be considered to uniquely represent a distinct piece of information (a piece of information different from the one that the physical quantity itself conveys which is that of its identity). Doing so is expected to bring advantage to computing.
\end{abstract}

Today, every piece of information is represented in computers by an appropriate sequence of two symbols, such as, 'True' and 'False', 'High' and 'Low' etc. [1]. Such a means of representation of information, called the binary system of representation of information, requires the use of a certain measure of space to store a certain amount of information. I, here, begin by proposing a means of representation of information and its storage which is supposed to reduce the use of space for storage of a certain amount of information by a great extent. By this means, a point in space is considered to uniquely represent one of two distinct pieces of information. We mark by some symbol the point in space corresponding to the piece of information we consider being stored by our computer. If we mark the point by a 'High' (one of the two symbols used in the binary system of representation of information), then the point is supposed to represent one the two abovementioned distinct pieces of information, and we say that that piece of information is being stored by our computer. If, on the other hand, we mark the point by a 'Low' (the other of the two symbols used in the binary system of representation of information), then the point is supposed to represent the other of the two abovementioned distinct pieces of information, and we say that this piece of information is being stored by our computer. This scheme enables us to store information of a certain amount in a measure of space which is much smaller than that required to store it using the binary system of representation of information. We illustrate this by an example in which we consider the problem of storage of 8 distinct pieces of information. In order to store 8 distinct pieces of information by means of the binary system of representation of information, we need to use $(8 \times 3)$ units of space, i.e., 24 units of space, where a unit of space is the measure of space required to store each of the two symbols used to represent information by this system. (We have assumed here that we need to use the same measure of space to store both the symbols used to represent information by this system.) This is because in order to represent and store any of these 8 pieces of information by this system, we require 3 units of space. But, to store these 8 distinct pieces of information by the means I have proposed here, we need to use 8 units of space, where a unit of space is the measure of space required to store each of the two symbols of the binary system of representation of information by which to mark a point in space used to represent any one of these 8 distinct pieces of information. Thus, to store 8 distinct pieces of information, we need to use space 
using the scheme of representation and storage of information I have proposed here of a measure which is one-third of the measure (of space) we need to use using the binary system of representation of information.

Let me elucidate the point. We consider the problem of storing 8 distinct pieces of information. Let us denote these 8 distinct pieces of information by I1, I2, I3, I4, I5, I6, I7 and I8.

Using the binary system of representation of information (the system of representation of information in which every piece of information is represented by an appropriate sequence of two symbols, such as, 'True' and 'False', 'High' and 'Low' etc.), these 8 distinct pieces of information may be represented as follows:

Information I1 may be represented as "Low Low Low".

Information I2 may be represented as "Low Low High".

Information I3 may be represented as "Low High Low".

Information I4 may be represented as "Low High High".

Information I5 may be represented as "High Low Low".

Information I6 may be represented as "High Low High".

Information $\mathrm{I} 7$ may be represented as "High High Low".

Information I8 may be represented as "High High High".

Hence, if we wish to store these 8 distinct pieces of information, I1, I2, I3, I4, I5, I6, I7 and I8, using the binary system of representation of information, we will require $(8 \times 3)$ units of space, i.e., 24 units of space, where a unit of space is the measure of space required to store each of the two symbols, 'High' and 'Low' used to represent information by this system. (We have assumed here that we need to use the same measure of space to store both the symbols, 'High' and 'Low', used to represent information by this system.) This is because in order to represent and store any of these 8 pieces of information by this system, we require 3 units of space, which is evident from the explanation regarding representing the 8 distinct pieces of information, I1, I2, I3, I4, I5, I6, I7 and I8, given above.

But, using the scheme of representing and storing information which I have proposed here, these 8 distinct pieces of information may be represented as follows:

Information I1 may be represented as the point P1 in space (say, the point $(0,0,0))$ when this point is marked by a 'High'.

Information I 2 may be represented as the point $\mathrm{P} 2$ in space (say, the point $(0,0,1))$ when this point is marked by a 'High'. 
Information I3 may be represented as the point P3 in space (say, the point $(0,1,0))$ when this point is marked by a 'Low'.

Information I4 may be represented as the point P4 in space (say, the point $(0,1,1))$ when this point is marked by a 'High'.

Information I5 may be represented as the point P5 in space (say, the point $(1,0,0))$ when this point is marked by a 'Low'.

Information I6 may be represented as the point P6 in space (say, the point $(1,0,1))$ when this point is marked by a 'Low'.

Information $\mathrm{I} 7$ may be represented as the point P7 in space (say, the point $(1,1,0)$ ) when this point is marked by a 'High'.

Information I8 may be represented as the point P8 in space (say, the point $(1,1,1))$ when this point is marked by a 'High'.

This is how these 8 pieces of information will be stored by our computer. Hence, in storing these 8 distinct pieces of information, I1, I2, I3, I4, I5, I6, I7 and I8, using the scheme of representing and storing information which I have proposed here, we will require 8 units of space, where a unit of space is the measure of space required to store the symbol 'High' (or the symbol 'Low').

We thus see that to store 8 distinct pieces of information, we need to use space using the scheme of representation and storage of information which I have proposed here of a measure which is one-third of the measure (of space) we need to use using the binary system of representation of information. Now, the $(24-8)$ units of space, i.e., 16 units of space that are free may be used to store any 16 out of $(16 \times 2)$ distinct pieces of information, i.e., 32 distinct pieces of information.

I also propose here the following means of representing information which are similar to the means of representing information proposed above (a point in space considered to uniquely represent a distinct piece of information (a piece of information different from the one that it itself conveys which is the information of its position)):

i) a colour can be considered to uniquely represent a distinct piece of information (a piece of information different from the one that the colour itself conveys which is the information of the colour),

ii) a certain frequency of an electromagnetic wave can be considered to uniquely represent a distinct piece of information (a piece of information different from the one that the frequency of the electromagnetic wave itself conveys which is the information of the frequency),

iii) a transition of a body (such as, an electron) from an energy state to another can be considered to uniquely represent a distinct piece of information (a piece of 
information different from the one that the transition itself conveys which is the information of the identity of the transition),

iv) energy of a certain magnitude can be considered to uniquely represent a distinct piece of information (a piece of information different from the one that the magnitude of the energy itself conveys which is the information of the magnitude of the energy),

v) a force can be considered to uniquely represent a distinct piece of information (a piece of information different from the one that the force itself conveys which is the information of the identity of the force),

vi) a potential can be considered to uniquely represent a distinct piece of information (a piece of information different from the one that the potential itself conveys which is the information of the identity of the potential),

vii) an atom of a kind can be considered to uniquely represent a distinct piece of information (a piece of information different from the one that the atom itself conveys which is the information of the identity of the atom),

viii) a molecule of a kind can be considered to uniquely represent a distinct piece of information (a piece of information different from the one that the molecule itself conveys which is the information of the identity of the molecule),

ix) a substance of a kind can be considered to uniquely represent a distinct piece of information (a piece of information different from the one that the substance itself conveys which is the information of the identity of the substance),

x) a magnitude of mass can be considered to uniquely represent a distinct piece of information (a piece of information different from the one that the magnitude of the mass itself conveys which is the information of the magnitude of the mass),

xi) a velocity can be considered to uniquely represent a distinct piece of information (a piece of information different from the one that the velocity itself conveys which is the information of the identity of the velocity),

xii) an acceleration can be considered to uniquely represent a distinct piece of information (a piece of information different from the one that the acceleration itself conveys which is the information of its identity),

xiii) a current can be considered to uniquely represent a distinct piece of information (a piece of information different from the one that the current itself conveys which is the information of the identity of the current),

xiv) a voltage can be considered to uniquely represent a distinct piece of information (a piece of information different from the one that the voltage itself conveys which is the information of the identity of the voltage), 
Xv) a pattern can be considered to uniquely represent a distinct piece of information (a piece of information different from the one that the pattern itself conveys which is the information of the identity of the pattern).

In fact, any physical quantity with a specific identity can be considered to uniquely represent a distinct piece of information (a piece of information different from the one that the physical quantity itself conveys which is that of its identity). Doing so is expected to bring advantage to computing.

\section{References:}

[1] M. Morris Mano, Computer Logic Design, Prentice-Hall, Inc., 1972. 